\title{
Combined effects of radiation and chemical reaction on MHD flow past a moving plate with Hall current
}

\author{
Uday Singh Rajput and Gaurav Kumar* \\ Department of Mathematics, Lucknow University, Lucknow(226007), U.P, INDIA. \\ "Corresponding Author: e-mail: rajputgauravlko@gmail.com, Tel +918574005045.
}

\begin{abstract}
Influence of radiation and chemical reaction on MHD flow past a moving plate with Hall current is studied here. Earlier, we (2016) have studied unsteady MHD flow in porous media over exponentially accelerated plate with variable wall temperature and mass transfer along with Hall current. To study further, we are changing the model by considering radiation and chemical reaction on flow, and changing geometry of the model. Now, we are taking the plate positioned vertically upward. Laplace method is used to solve the flow model. The results obtained have been analyzed with the help of graphs drawn for different parameters. The numerical values obtained for the drag, Sherwood and Nusselt numbers at boundary have been tabulated. Here too, the results are found to be in agreement with the actual flow.
\end{abstract}

Keywords: MHD flow, radiation, chemical reaction.

DOI: http://dx.doi.org/10.4314/ijest.v9i4.6

\section{Introduction}

MHD flow problems over an impulsively started vertical plate play important role in many branches of science and technology. The effects of radiation and chemical reaction on MHD flow are also significant in many cases. Some such problems already studied are mentioned here. The Hall effect in the viscous flow of ionized gas between parallel plates under transverse magnetic field was studied by Sato (1961). Mazumder and Deka (2007) have considered MHD flow past an impulsively started infinite vertical plate in the presence of thermal radiation. Ibrahim and Makinde (2010) have investigated chemically reacting MHD boundary layer flow of heat and mass transfer over a moving vertical plate with suction. Reddy et al (2014) have studied MHD flow considering free convection over a porous plate. Balla and Naikoti (2015) have considered unsteady MHD flow with convective heat and mass diffusion. MHD flow over a stretching surface was analyzed by Jonnadula et al (2015). Malapati and Polarapu (2015) have worked on MHD flow with natural convection. Unsteady MHD flow in porous media was investigated by us (2016). Rajput and Kanaujia (2016) have worked on chemical reaction in MHD flow past a vertical plate with mass diffusion and constant wall temperature with Hall current. The motive of this study is to analyze the combined effects of radiation and chemical reaction on fluid flow over a moving plate in the presence of transversely applied uniform magnetic field and Hall current. The fluid model under consideration has been solved by Laplace transform method. The model contains equations of motion, diffusion equation and equation of energy. To analyze the solution of the model, desirable sets of the values of the parameters have been considered. The numerical data obtained is discussed with the help of graphs and tables.

\section{Mathematical Analysis}

The physical model is shown in Figure-1 


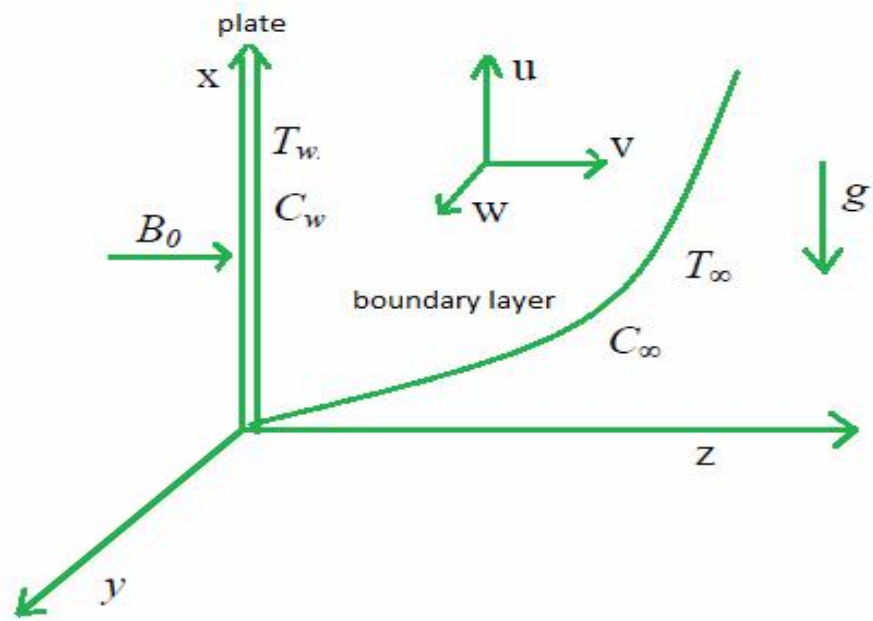

Figure-1

Consider an unsteady MHD flow past an impulsively started vertical plate. The fluid is electrically conducting. The $\mathrm{x}$ axis is along the vertical plate and $\mathrm{z}$ is perpendicular to it. Thus the $\mathrm{z}$ axis lies in the horizontal plane. The uniform magnetic field $B_{0}$ is applied perpendicular to the fluid. Initially it has been considered that the plate as well as the fluid is at the same temperature $T_{\infty}$. The species concentration in the fluid is taken as $C_{\infty}$. At time $\mathrm{t}>0$, the plate starts moving with a velocity $\mathrm{u}_{0}$. The wall temperature $T_{w}$ and the concentration $C_{w}$ in the boundary region are raised in proportion with time. So, under above assumptions, the governing equations are as follows:

Momentum Equation

$$
\begin{aligned}
& \frac{\partial u}{\partial t}=v \frac{\partial^{2} u}{\partial z^{2}}+g \beta\left(T-T_{\infty}\right)+g \beta^{*}\left(C-C_{\infty}\right)-\frac{\sigma B_{0}^{2}(u+m v)}{\rho\left(1+m^{2}\right)} \\
& \frac{\partial v}{\partial t}=v \frac{\partial^{2} v}{\partial z^{2}}+\frac{\sigma B_{0}^{2}(m u-v)}{\rho\left(1+m^{2}\right)}
\end{aligned}
$$

Concentration Equation

$\frac{\partial C}{\partial t}=D \frac{\partial^{2} C}{\partial z^{2}}-K_{c}\left(C-C_{\infty}\right)$

Energy Equation

$\rho C_{p} \frac{\partial T}{\partial t}=k \frac{\partial^{2} T}{\partial z^{2}}-\frac{\partial q_{r}}{\partial z}$

Here $K_{c}$ is chemical parameter, $u$ and $v$ are the primary and secondary velocities along $\mathrm{x}$ and $\mathrm{z}$ directions respectively.

Other symbols used have the following description:

$C$ - species concentration in the fluid, $\mathrm{m}$-the Hall current parameter, $T$-temperature of the fluid, $T_{w}$ - temperature of the plate at $z=$

$0, C_{w}$-species concentration at the plate $z=0, B_{0^{-}}$the uniform magnetic field, $\sigma$ - electrical conductivity. $\beta^{*}$ - volumetric

coefficient of concentration expansion, $\nu$ - the kinematic viscosity, $\rho$ - the density, $C_{p^{-}}$- the specific heat at constant pressure, $g$-the

acceleration due to gravity, $\beta$-volumetric coefficient of thermal expansion, $t$-time, $k$-thermal conductivity of the fluid, $D$-the mass diffusion coefficient.

The boundary conditions taken are as under:

$\left.\begin{array}{l}t \leq 0: u=v=0, T=T_{\infty}, C=C_{\infty}, \text { for every } z \\ t>0: u=u_{0}, v=0, T=T+\left(T_{w}-T_{\infty}\right) A, C=C_{\infty}+\left(C_{w}-C_{\infty}\right) A, \text { at } z=0 .\end{array}\right\}$ 
$u \rightarrow 0, v \rightarrow 0, T \rightarrow T_{\infty}, C \rightarrow C_{\infty}$ as $z \rightarrow \infty$

By using Rosseland approximation (Brewster (1992)), the radiative heat flux $q_{r}$ is given by

$\frac{\partial q_{r}}{\partial z}=-4 a^{*} \sigma\left(T_{\infty}^{4}-T^{4}\right)$

where $a^{*}$ is absorption constant.

The temperature difference in the flow is considered sufficiently small, therefore $T^{4}$ can be approximated by Taylor series. Hence $T^{4} \cong 4 T_{\infty}^{3} T-3 T_{\infty}^{4}$

Energy equation (4) is transformed under equations (6) and (7), which is as below:

$\rho C_{p} \frac{\partial T}{\partial t}=k \frac{\partial^{2} T}{\partial z^{2}}-16 a^{*} \sigma T_{\infty}^{3}\left(T-T_{\infty}\right)$

To transform equations (1), (2), (3) and (8) into dimensionless form, we use the following quantities:

$\left.\begin{array}{l}\bar{z}=\frac{z u_{0}}{v}, \bar{u}=\frac{u}{u_{0}}, \bar{v}=\frac{v}{u_{0}}, \theta=\frac{\left(T-T_{\infty}\right)}{\left(T_{w}-T_{\infty}\right)}, S_{c}=\frac{v}{D}, \quad=\rho v, \bar{C}=\frac{\left(C-C_{\infty}\right)}{\left(C_{w}-C_{\infty}\right)}, R=\frac{16 a^{*} v^{2} \sigma T_{\infty}^{3}}{k u_{0}} \\ G_{r}=\frac{g \beta v\left(T_{w}-T_{\infty}\right)}{u_{0}^{3}}, M=\frac{\sigma B_{0}^{2} v}{\rho u_{0}^{2}}, G_{m}=\frac{g \beta^{*} v\left(C_{w}-C_{\infty}\right)}{u_{0}^{3}}, K_{0}=\frac{v K_{c}}{u_{0}^{2}} P_{r}=\frac{u_{0}}{k}, \bar{t}=\frac{t u_{0}^{2}}{v} .\end{array}\right\}$

The symbols in dimensionless form are as under:

$K_{0}$ - chemical reaction, $R$ - Radiation parameter, $\bar{u}$ - primary velocity, $\bar{v}$ - secondary velocity, $P_{r}$ - Prandtl number, $S_{c}$ - Schmidt number, $\bar{t}$ - time, $\theta$ - temperature, $\bar{C}$ - concentration, $G_{r}$ - thermal Grashof number, $G_{m}$ - mass Grashof number, - coefficient of viscosity, $M$ - magnetic parameter.

The flow model in dimensionless form is

$$
\begin{aligned}
& \frac{\partial \bar{u}}{\partial \bar{t}}=\frac{\partial^{2} \bar{u}}{\partial \bar{z}^{2}}+G_{r} \theta+G_{m} \bar{C}-\frac{M(\bar{u}+m \bar{v})}{\left(1+m^{2}\right)} \\
& \frac{\partial \bar{v}}{\partial \bar{t}}=\frac{\partial^{2} \bar{u}}{\partial \bar{z}^{2}}+\frac{M(m \bar{u}-\bar{v})}{\left(1+m^{2}\right)} \\
& \frac{\partial \bar{C}}{\partial \bar{t}}=\frac{1}{S_{c}} \frac{\partial^{2} \bar{C}}{\partial \bar{z}^{2}}-K_{0} \bar{C} \\
& \frac{\partial \theta}{\partial \bar{t}}=\frac{1}{P_{r}} \frac{\partial^{2} \theta}{\partial \bar{z}^{2}}-\frac{R \theta}{P_{r}}
\end{aligned}
$$

The boundary conditions (5) become:

$$
\left.\begin{array}{l}
\bar{t} \leq 0: \bar{u}=\bar{v}=\theta=\bar{C}=0, \quad \text { for every } \bar{z} . \\
\bar{t}>0: \bar{u}=1, \bar{v}=0, \theta=\bar{t}, \bar{C}=\bar{t}, \quad \text { at } \quad \bar{z}=0 . \\
\bar{u} \rightarrow 0, \bar{v} \rightarrow 0, \theta \rightarrow 0, \bar{C} \rightarrow 0, \quad \text { as } \quad \bar{z} \rightarrow \infty .
\end{array}\right\}
$$

Removing bars, for simplicity, we get

$$
\frac{\partial u}{\partial t}=\frac{\partial^{2} u}{\partial z^{2}}+G \theta+G_{m} C-\frac{M(u+m v)}{\left(1+m^{2}\right)}
$$




$$
\begin{aligned}
& \frac{\partial v}{\partial t}=\frac{\partial^{2} v}{\partial z^{2}}+\frac{M(m u-v)}{\left(1+m^{2}\right)} \\
& \frac{\partial C}{\partial t}=\frac{1}{S_{c}} \frac{\partial^{2} C}{\partial z^{2}}-K_{0} C \\
& \frac{\partial \theta}{\partial t}=\frac{1}{P_{r}} \frac{\partial^{2} \theta}{\partial z^{2}}-\frac{R \theta}{P_{r}}
\end{aligned}
$$

$t \leq 0: u=v=\theta=C=0, \quad$ for every $z$

$t>0: u=1, v=0, \theta=t, C=t, \quad$ at $\mathrm{z}=0$.

$u \rightarrow 0, v \rightarrow 0, \theta \rightarrow 0, C \rightarrow 0, \quad$ as $z \rightarrow \infty$.

Writing the equations (15) and (16) in combined form (using $q=u+i v$ )

$$
\begin{aligned}
& \frac{\partial q}{\partial t}=\frac{\partial^{2} q}{\partial z^{2}}+G_{r} \theta+G_{m} C-q a \\
& \frac{\partial C}{\partial t}=\frac{1}{S_{c}} \frac{\partial^{2} C}{\partial z^{2}}-K_{0} C \\
& \frac{\partial \theta}{\partial t}=\frac{1}{P_{r}} \frac{\partial^{2} \theta}{\partial z^{2}}-\frac{R \theta}{P_{r}}
\end{aligned}
$$

The boundary conditions (19) are reduced to:

$\left.\begin{array}{l}t \leq 0: q=\theta=C=0, \quad \text { for every z. } \\ t>0: q=1, \theta=t, C=t, \quad \text { at } \quad \mathrm{z}=0 . \\ q \rightarrow 0, \theta \rightarrow 0, C \rightarrow 0, \quad \text { as } z \rightarrow \infty .\end{array}\right\}$

The equations (20) to (22), using equation (23), are solved by the Laplace method. The solution obtained is as under:

$$
\begin{aligned}
q= & \frac{1}{2} \exp (-\sqrt{a} z) A_{33}+\frac{G_{r}}{4(a-R)^{2}}\left(\left(\exp (-\sqrt{a} z)\left(2 R t A_{1}-2 a t A_{1}+z \sqrt{a} A_{2}+2 A_{1}\left(P_{r}+1\right)\right)-\frac{A_{2} z}{\sqrt{a}}\right)-\frac{2 A_{5} P_{r} z}{\sqrt{A_{32}} A_{11}}(a t-R t\right. \\
& \left.\left.+P_{r}-1\right)+\frac{2 A_{28} A_{6} P_{r} z}{A_{11}}\left(P_{r}-1\right)-2 A_{26} A_{3}\left(P_{r}-1\right)-\frac{P_{r} z \sqrt{A_{32} P_{r}}}{A_{10} \pi \sqrt{R}}\left(\frac{1}{a}-\frac{1}{R}\right)\right)+\frac{G_{m}}{4\left(a-K_{0} S_{c}\right)^{2}}\left(\left(\operatorname { e x p } ( - \sqrt { a } z ) \left(z \sqrt{a} A_{2}\right.\right.\right. \\
& \left.\left.-2 a t A_{1}-2 A_{1}\left(S_{c}-1\right)+2 t A_{1} K_{0} S_{c}\right)-\frac{z \exp (-\sqrt{a} z) A_{2} K_{0} S_{c}}{\sqrt{a}}-2 A_{27} A_{4}\left(S_{c}-1\right)\right)+\exp \left(-z \sqrt{S_{c} K_{0}}\right)\left(-\frac{a A_{8} z \sqrt{S_{c}}}{\sqrt{K_{0}}}\right. \\
& \left.\left.-2 a t A_{7}-2 A_{7}-2 A_{7}\left(S_{c}-1\right)+2 t A_{7} K_{0} S_{c}+z A_{8} S_{c} \sqrt{S_{c} K_{0}}\right)+2 A_{27} A_{9}\left(S_{c}-1\right)\right) . \\
\theta= & \frac{\mathrm{e}^{-\sqrt{\mathrm{R}} z}}{4 \sqrt{R}}\left\{\operatorname{erfc}\left[\frac{-2 \sqrt{R} t+z P_{r}}{\sqrt{P_{r} \mathrm{t}}}\right](2 \sqrt{R} t-z R)+\mathrm{e}^{2 \sqrt{\mathrm{R}} z} \operatorname{erfc}\left[\frac{2 \sqrt{R} t+z P_{r}}{\sqrt{P_{r} \mathrm{t}}}\right](2 \sqrt{R} t+z R)\right\}, \\
C= & \frac{e^{-z \sqrt{S_{c} K_{0}}}}{4 \sqrt{K_{0}}}\left\{\operatorname{erfc}\left[\frac{z \sqrt{S_{c}}-2 t \sqrt{K_{0}}}{2 \sqrt{t}}\right]\left(-z \sqrt{S_{c}}+2 t \sqrt{K_{0}}\right)+e^{2 z \sqrt{S_{c} K_{0}}} \operatorname{erfc}\left[\frac{z \sqrt{S_{c}}+2 t \sqrt{K_{0}}}{2 \sqrt{t}}\right]\left(z \sqrt{S_{c}}+2 t \sqrt{K_{0}}\right)\right\} .
\end{aligned}
$$

The expressions for the symbols involved in the above solution are given in the appendix.

\section{Skin Friction}


The dimensionless skin-friction at the surface is

$$
\left(\frac{d q}{d z}\right)_{z=0}=\tau_{x}+i \tau_{y} .
$$

The numerical values of $\tau_{x}$ and $\tau_{y}$, for different parameters, are given in table-1.

\section{Nusselt number}

The dimensionless Nusselt number is given by

$$
N u=\left(\frac{\partial \theta}{\partial z}\right)_{z=0}=\operatorname{erfc}\left[\frac{\sqrt{R} t}{\sqrt{t P_{r}}}\right]\left(\sqrt{R} t-\frac{\sqrt{R}}{2} t+\frac{P_{r}}{4 \sqrt{R}}\right)-\operatorname{erfc}\left[-\frac{\sqrt{R} t}{\sqrt{t P_{r}}}\right]\left(\frac{\sqrt{R}}{2} t+\frac{P_{r}}{4 \sqrt{R}}\right)-\frac{e^{-\frac{R t}{P_{r}}} \sqrt{t P_{r}}}{\sqrt{\pi}} .
$$

\section{Sherwood number}

The dimensionless Sherwood number at the surface is

$$
S_{h}=\left(\frac{\partial C}{\partial z}\right)_{z=0}=\operatorname{erfc}\left[-\sqrt{t K_{0}}\right]\left(-\frac{1}{4 \sqrt{K_{0}}} \sqrt{S_{c}}-\frac{t \sqrt{S_{c} K_{0}}}{2}\right)+\sqrt{S_{c}} \operatorname{erfc}\left[\sqrt{t K_{0}}\right]\left(\frac{1}{4 \sqrt{K_{0}}}+t \sqrt{K_{0}}\right)-\frac{e^{-t K_{0}} \sqrt{t S_{c} K_{0}}}{\sqrt{\pi K_{0}}}
$$

\section{Results and discussion}

The study is carried out to examine the effects of radiation with chemical reaction on unsteady MHD flow past a moving plate with variable wall temperature and mass diffusion in the presence of Hall current. The behavior of other parameters like magnetic parameter, Hall current and thermal buoyancy is almost similar to the earlier model studied by us (2016). The analytical results are shown in figures 2 to 7 . The numerical values of skin-friction, Sherwood number and Nusselt number are presented in Table-1, 2 and 3, respectively. Chemical reaction effect on fluid flow behavior is shown by figures 2 and 3. It is seen here, when chemical reaction parameter $\mathrm{K}_{0}$ increases, $u$ and $v$ decrease throughout near the surface. Figures 4 and 5 indicate that effect of radiation in the flow near the plate tends to accelerate velocities. This is due to the fact that the large values of radiation parameter tend to accelerate velocity of the fluid in the region near the surface of the plate. Further, it is noticed that the temperature and concentration of the fluid near the plate decrease when radiation and chemical reaction parameters are increased (figures 6 and 7).

Skin friction is given in table 1 . The values of Skin friction $\tau_{x}$ and $\tau_{y}$ increase with the increase in $R$ and decrease with $K_{o}$. Sherwood number is given in table 2. The value of $S_{h}$ decreases with the increase in $K_{o}, S_{c}$ and $t$. Nusselt number is given in table 3. The value of $N u$ decreases with increase in $P_{r}, R$ and $t$.

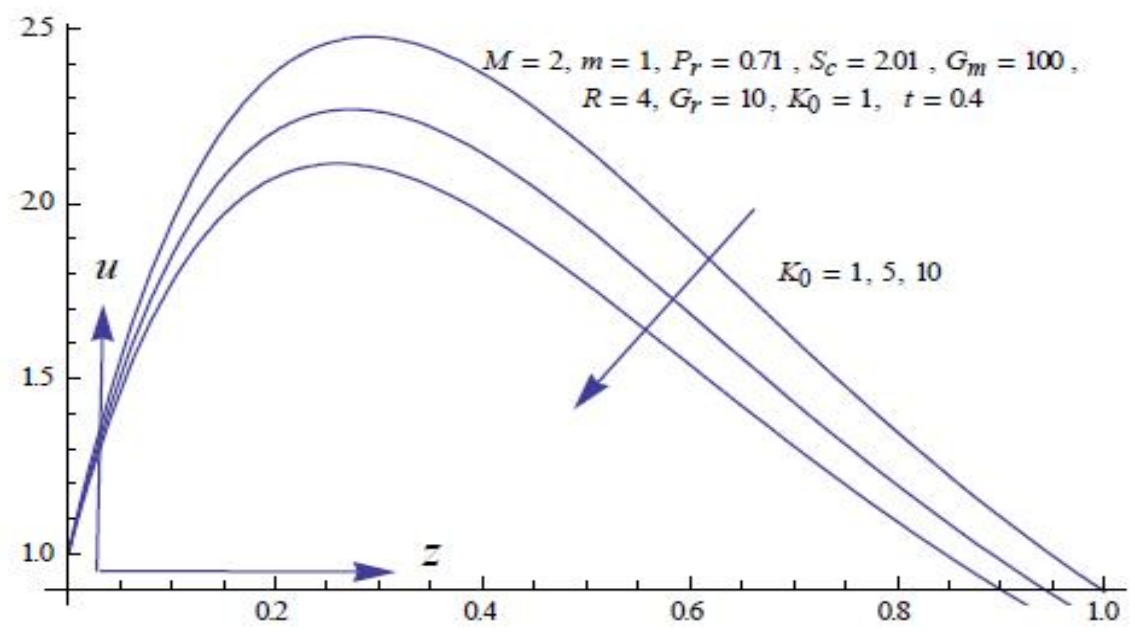

Figure 2: $u v s z$ 


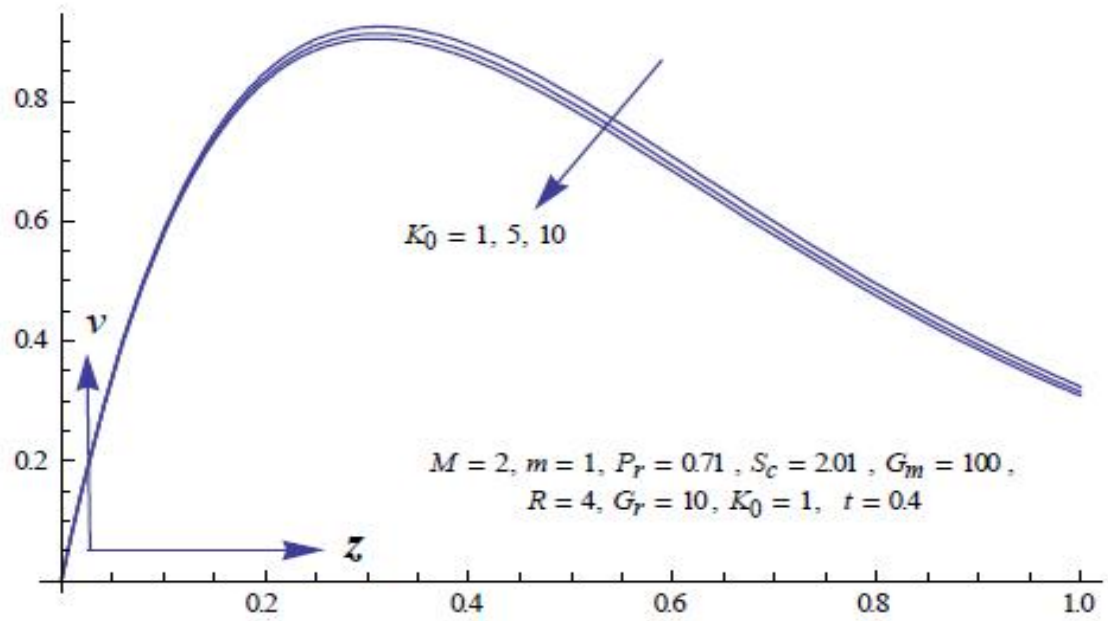

Figure 3: $v v s z$

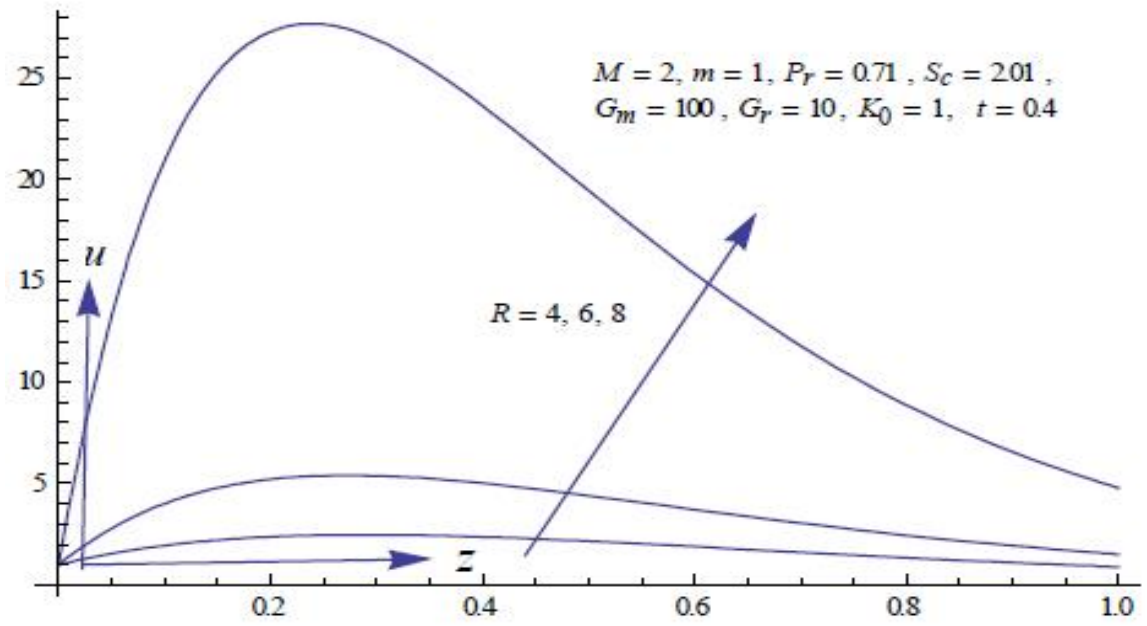

Figure 4: $u v s z$

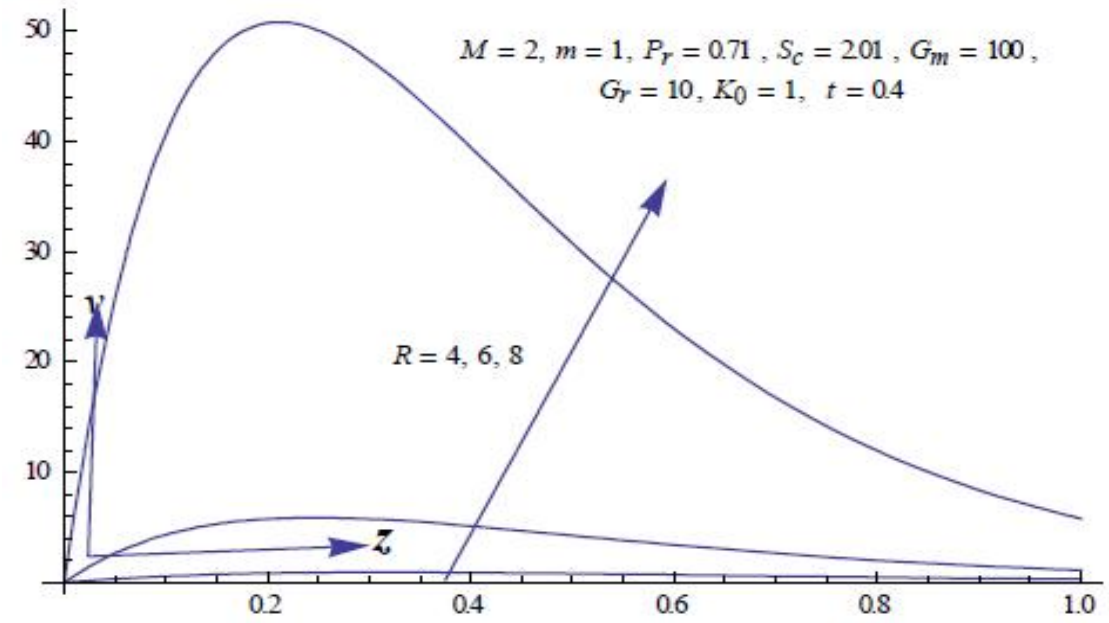

Figure 5: $v$ vs $z$ 


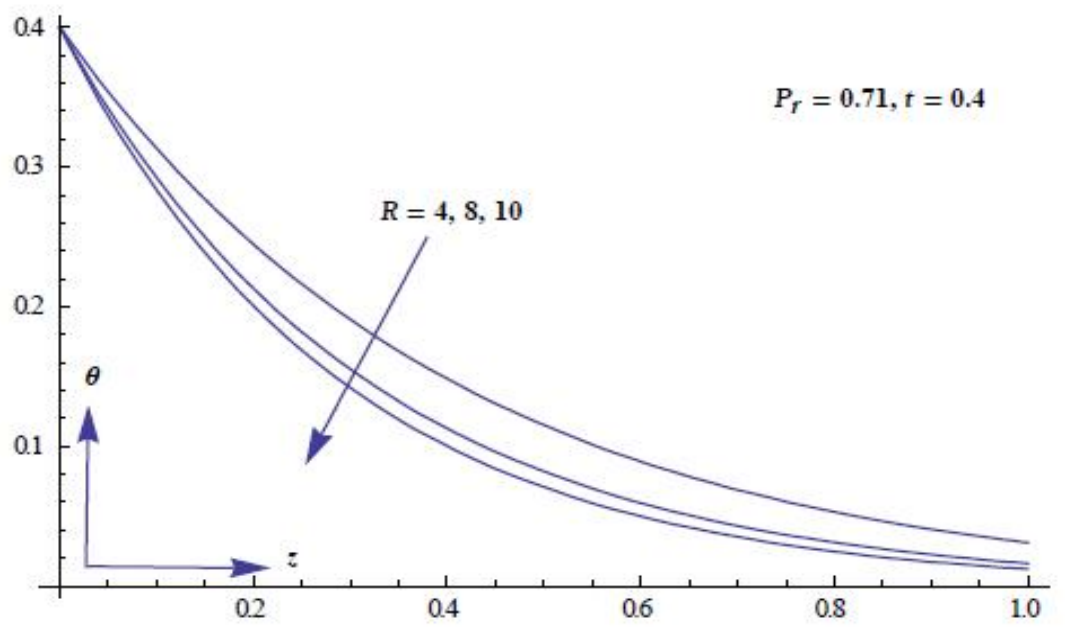

Figure 6: $\theta$ vs $z$

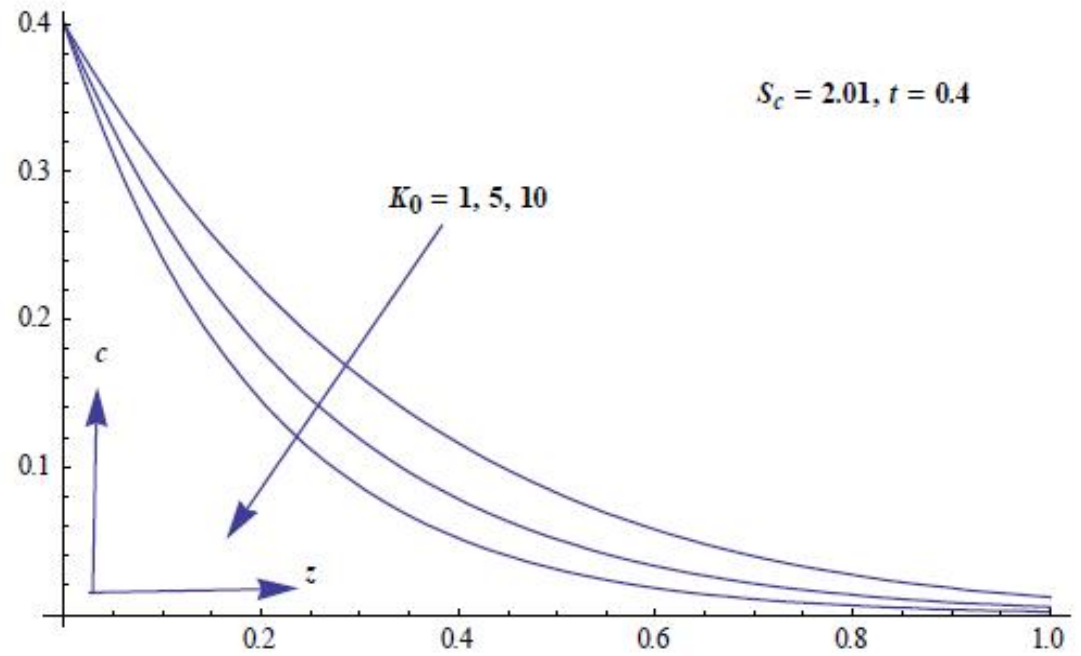

Figure 7: $c$ vs $z$

Table 1: Skin-friction for different parameters.

\begin{tabular}{|c|c|c|c|c|c|c|c|c|c|c|}
\hline$M$ & $m$ & $P r$ & $S c$ & $G m$ & $G r$ & $R$ & $K_{0}$ & $t$ & & \\
\hline 2 & 1 & 0.71 & 2.01 & 100 & 10 & 04 & 01 & 0.4 & 01215.7783 & 01284.2268 \\
\hline 2 & 1 & 0.71 & 2.01 & 100 & 10 & 06 & 01 & 0.4 & 06052.4816 & 09261.1973 \\
\hline 2 & 1 & 0.71 & 2.01 & 100 & 10 & 08 & 01 & 0.4 & 41596.3813 & 80992.4326 \\
\hline 2 & 1 & 0.71 & 2.01 & 100 & 10 & 04 & 05 & 0.4 & 01214.7722 & 01248.1817 \\
\hline 2 & 1 & 0.71 & 2.01 & 100 & 10 & 04 & 10 & 0.4 & 01213.9525 & 01248.1458 \\
\hline
\end{tabular}

Table2. Sherwood number for different Parameters.

\begin{tabular}{|c|c|c|c|}
\hline$K_{0}$ & $\mathrm{Sc}$ & $\mathrm{T}$ & $S_{h}$ \\
\hline 01 & 2.01 & 0.2 & -0.762200 \\
\hline 05 & 2.01 & 0.2 & -0.933049 \\
\hline 10 & 2.01 & 0.2 & -1.118240 \\
\hline
\end{tabular}




\begin{tabular}{|l|l|l|l|}
\hline 01 & 3.00 & 0.2 & -0.931175 \\
\hline 01 & 4.00 & 0.2 & -1.075230 \\
\hline 01 & 2.01 & 0.3 & -0.961323 \\
\hline 01 & 2.01 & 0.4 & -1.141570 \\
\hline
\end{tabular}

Table 3: Nusselt number for different parameters

\begin{tabular}{|c|c|c|c|}
\hline$P r$ & $R$ & $t$ & $N u$ \\
\hline 0.71 & 2 & 0.4 & -0.805273 \\
\hline 7.00 & 2 & 0.4 & -1.959260 \\
\hline 0.71 & 3 & 0.4 & -0.894014 \\
\hline 0.71 & 4 & 0.4 & -0.976083 \\
\hline 0.71 & 2 & 0.5 & -0.950956 \\
\hline 0.71 & 2 & 0.6 & -1.094940 \\
\hline
\end{tabular}

\section{Conclusion}

The results obtained are in agreement with the usual flow. It is observed that the velocities near the plate surface are increased with radiation parameter, and decrease with chemical reaction. Further, due to the radiation the drag at the plate surface increases. However the drag decreases with chemical reaction. Also the values of $S_{h}$ and $N_{u}$ decrease with $K_{0}$ and $R$. It is as per expected flow behavior.

\section{Appendix:}

$$
\begin{aligned}
& a=\frac{M(1-i m)}{1+m^{2}}, A=\frac{u_{0}^{2} t}{v}, A_{1}=\left(1+A_{12}+e^{2 \sqrt{a z}}\left(1-A_{13}\right)\right), A_{2}=\left(1+A_{12}-e^{2 \sqrt{a z}}\left(1-A_{13}\right)\right), \\
& A_{3}=\left(A_{14}-1+A_{29}\left(A_{15}-1\right)\right), A_{4}=\left(A_{16}-1+A_{30}\left(A_{17}-1\right)\right), A_{5}=\left(A_{18}-1+A_{33}\left(A_{19}-1\right)\right), \\
& \left.\left.A_{6}=\left(A_{20}-1+A_{31}\left(A_{21}-1\right)\right), A_{7}=\left(e^{2 z \sqrt{K_{0} S_{c}}}\left(A_{23}-1\right)-A_{22}-1\right)\right), A_{8}=\left(e^{2 z \sqrt{K_{0} S_{c}}}\left(A_{23}-1\right)+A_{22}+1\right)\right), \\
& A_{9}=\left(A_{30}\left(A_{25}-1\right)-A_{24}-1\right), A_{10}=\left(1-A_{18}+A_{32}\left(A_{19}-1\right)\right), A_{11}=A b s[z] A b s\left[P_{r}\right], A_{12}=\operatorname{erf}\left[\frac{1}{2 \sqrt{t}}(2 \sqrt{a} t-z)\right], \\
& A_{13}=\operatorname{erf}\left[\frac{1}{2 \sqrt{t}}(2 \sqrt{a} t+z)\right], A_{14}=\operatorname{erf}\left[\frac{1}{2 \sqrt{t}}\left(z-2 t \sqrt{\frac{a P_{r}-R}{P_{r}-1}}\right)\right], A_{15}=\operatorname{erf}\left[\frac{1}{2 \sqrt{t}}\left(z+2 t \sqrt{\frac{a P_{r}-R}{P_{r}-1}}\right)\right], \\
& A_{16}=\operatorname{erf}\left[\frac{1}{2 \sqrt{t}}\left(z-2 t \sqrt{\frac{\left(a-K_{0}\right) S_{c}}{S_{c}-1}}\right)\right], A_{17}=\operatorname{erf}\left[\frac{1}{2 \sqrt{t}}\left(z+2 t \sqrt{\frac{\left(a-K_{0}\right) S_{c}}{S_{c}-1}}\right)\right], A_{18}=\operatorname{erf}\left[\frac{A_{11}}{2 \sqrt{t}}-\sqrt{\frac{t R}{P_{r}}}\right] \text {, } \\
& \left.\left.A_{19}=\operatorname{erf}\left[\frac{A_{11}}{2 \sqrt{t}}+\sqrt{\frac{t R}{P_{r}}}\right], A_{20}=\operatorname{erf}\left[\frac{A_{11}}{2 \sqrt{t}}-\sqrt{\frac{\left(R-a P_{r}\right) t}{P_{r}-P_{r}^{2}}}\right)\right], A_{21}=\operatorname{erf}\left[\frac{A_{11}}{2 \sqrt{t}}+\sqrt{\frac{\left(R-a P_{r}\right) t}{P_{r}-P_{r}^{2}}}\right)\right], \\
& A_{22}=\operatorname{erf}\left[\frac{1}{2 \sqrt{t}}\left(2 t \sqrt{K_{0}}-z \sqrt{S_{c}}\right)\right], A_{23}=\operatorname{erf}\left[\frac{1}{2 \sqrt{t}}\left(2 t \sqrt{K_{0}}+z \sqrt{S_{c}}\right)\right], A_{24}=\operatorname{erf}\left[\frac{1}{2 \sqrt{t}}\left(2 t \sqrt{\frac{\left(a-K_{0}\right) S_{c}}{S_{c}-1}}-z \sqrt{S_{c}}\right)\right] \text {, } \\
& A_{25}=\operatorname{erf}\left[\frac{1}{2 \sqrt{t}}\left(2 t \sqrt{\frac{\left(a-K_{0}\right) S_{c}}{S_{c}-1}}+z \sqrt{S_{c}}\right)\right], A_{26}=\exp \left(\frac{a t}{P_{r}-1}-\frac{R t}{P_{r}-1}-z \sqrt{\frac{a P_{r}-R}{P_{r}-1}}\right), \\
& A_{27}=\exp \left(\frac{a t}{S_{c}-1}-\frac{t S_{c} K_{0}}{S_{c}-1}-z \sqrt{\frac{\left(a-K_{0}\right) S_{c}}{S_{c}-1}}\right), A_{28}=\frac{1}{A_{31}} \exp \left(\frac{a t}{P_{r}-1}-\frac{R t}{P_{r}-1}\right), A_{29}=\exp \left(2 z \sqrt{\frac{-R+a P_{r}}{P_{r}-1}}\right), \\
& A_{30}=\exp \left(2 z \sqrt{\frac{\left(a-K_{0}\right) S_{c}}{S_{c}-1}}\right) \text {, }
\end{aligned}
$$




$$
\begin{aligned}
& A_{31}=\exp \left(2 A b s[z] \sqrt{\frac{P_{r}\left(a P_{r}-R\right)}{P_{r}-1}}\right), A_{32}=\exp \left(2 A b s[z] \sqrt{P_{r} R}\right), A_{33}=1+A_{34}+\exp (2 \sqrt{a z}) A_{35}, \\
& A_{34}=\operatorname{erf}\left[\frac{1}{2 \sqrt{t}}(2 \sqrt{a} t-z)\right], A_{35}=\operatorname{erfc}\left[\frac{1}{2 \sqrt{t}}(2 \sqrt{a} t+z)\right],
\end{aligned}
$$

\section{References}

Balla C. S. and Naikoti K., 2015. Radiation effects on unsteady MHD convective heat and mass transfer past a vertical plate with chemical reaction and viscous dissipation. Alexandria Engineering Journal, Vol. 54, pp 661-671.

Brewster M.Q., 1992. Thermal Radiative Transfer and Properties. John Wiley \& Sons, New York.

Ibrahim S. Y., and Makinde O. D., 2010. Chemically reacting MHD boundary layer flow of heat and mass transfer over a moving vertical plate with suction. Scientific Research and Essays, Vol. 5, No. 19, pp. 2875-2882.

Jonnadula M., Polarapu P., Reddy M. G. and Venakateswarlu M., 2015. Influence of thermal radiation and chemical reaction on MHD flow, heat and mass transfer over a stretching surface. International Conference on Computational Heat and Mass Transfer, Procedia Engineering (Elsevier), Vol. 127, pp. 1315 - 1322.

Malapati V. and Polarapu P., 2015. Unsteady MHD free convection heat and mass transfer in a boundary layer flow past a vertical permeable plate with thermal radiation and chemical reaction. Procedia Engineering, 127, pp $791-799$.

Mazumder M. and Deka R. K., 2007. MHD flow past an impulsively started infinite vertical plate in presence of thermal radiation, Romanian Journal of Physics, Vol. 52, No. (5-6), pp 529-535.

Rajput U.S. and Kumar G., 2016. Unsteady MHD flow in porous media past over exponentially accelerated inclined plate with variable wall temperature and mass transfer along with Hall current. International Journal of Engineering, Science and Technology, Vol. 8, No. 2, pp. 01-10.

Rajput U. S. and Kanaujia N., 2016. Chemical reaction in MHD flow past a vertical plate with mass diffusion and constant wall temperature with hall current. International Journal of Engineering, Science and Technology Vol. 8, No. 4, pp. 28-38.

Reddy N. A , Raju M.C. and Varma S.V.K., 2014. Analytical study of MHD free convective, dissipative boundary layer flow past a porous vertical surface in the presence of thermal radiation, chemical reaction and constant suction. Ain Shams Engineering Journal, Vol. 5, pp 1361-1369.

Sato H., 1961. The Hall effect in the viscous flow of ionized gas between parallel plates under transverse magnetic field. Journal of the Physical Society of Japan, Vol. 16, No. 7, pp 1427-1433.

\section{Biographical notes}

Dr Uday Singh Rajput is a faculty member in the department of mathematics and astronomy, Lucknow University, India. He has more than 25 years of teaching experience at UG and PG levels and also guided students for $\mathrm{PhD}$ degree. He has published more than 70 research articles. His research areas include MHD flows, Graph Theory and Operations Research.

Gaurav Kumar is research student in the department of mathematics and astronomy, Lucknow University, India.

Received June 2016

Accepted July 2017

Final acceptance in revised form August 2017 